\title{
PHYSICAL THERAPY IN RHEUMATOID ARTHRITIS
}

\author{
James W. Rae, Jr., M.D., and Leonard F. Bender, M.D. \\ ANN ARbor, Mich.
}

\begin{abstract}
From the Department of Physical Medicine and Rehabilitation, Medical School, University Hospital, University of Michigan
\end{abstract}

(Received for publication Feb. 5, 1957.)

\begin{abstract}
$I^{\mathrm{N}}$ SPITE of new discoveries and advances in the treatment of rheumatoid arthritis, the most important part of the management of patients with this disease today is the prevention or minimizing of deformity and the maintenance or improvement of function. The numerous remedies that have been promoted for use in arthritis tend to detract from the appreciation of the value of physical treatment in preventing crippling and disability. Hill and $\mathrm{Holbrook}^{1}$ have estimated that not one patient in 100 is following a proper schedule to protect his joints and maintain function. This is a serious indictment of our medical care programs for patients with rheumatoid arthritis. Whether one agrees with these figures or not, all will agree that a high percentage of patients fail to carry out any program, or go at it halfheartedly, or eventually tend to lose interest in even a carefully planned and effective schedule of treatment.

A review of the literature from 1940 to the present offers little information as to the best way to motivate a patient with a chronic ailment to treat himself over a long period of time. In 1943, a report from the Mayo $\mathrm{Clinic}^{2}$ gave the results of a survey of arthritis patients chosen at random from several thousand clinic patients who had been instructed in home physical therapy. Of the 346 patients to whom the investigators sent a questionnaire, 216 responded, and the results showed that 92.7 per cent had started the treatment program and 64.7 per cent had continued treatment for three months or more. There were 96 patients with rheumatoid arthritis in this group; 63.5 per cent of these were continuing home treatment or else had stopped because their condition had improved.
\end{abstract}

A survey of arthritis patients at University Hospital was recently conducted to help evaluate our program of instruction in home treatment and shed some light on why patients continue or stop their home treatment schedules. Fiftysix patients who had previously been instructed in adequate home programs were personally interviewed. At the time of the interview, 5 patients had stopped their home treatment because of improvement in their condition. Of the remaining 51 patients, 27 were still continuing adequate home treatment programs, but 24 patients who needed physical therapy were not treating themselves adequately. 
Six of these 24 patients never started on their recommended home program; 8 stopped home treatment between 2 and 4 months after thcir instruction. This was greater than the number stopping in any other comparable period of time.

There are various factors contributing to the frequent failure to adequately utilize physical treatment in the management of rheumatoid arthritis. Some of these are personal concerns of the patient, situations in which the physician may offer counsel but which otherwise are beyond his control. But even the. most ideally situated and motivated patient will fail in treatment if his physician has failed to understand and convey the importance of established principles of care, or has provided inadequate prescription of physical measures, improper scheduling of activities, inadequate instruction of the patient, or inadequate follow-up care and evaluation.

Basic Principles of Care.-Every physician who assumes the responsibility of caring for the patient with rheumatoid arthritis should recognize the need for establishing a program of symptomatic, constitutional, and supportive measures designed to relieve pain, prevent or minimize deformity, preserve or increase joint range and muscle strength, and maintain or improve functional capacity. Physical treatment consisting of heat, massage, and therapeutic exercise has proved valuable in attaining these goals. To be most effective, physical therapy must be combined with the other established principles of care such as increased general rest, adequate local rest of the involved joints, avoidance of strain and irritation of the involved joints, intelligent use of splints, supports, shoes, and other medical agents (steroids, gold salts, etc.), and surgical procedures as indicated. Daily home treatment prescribed and supervised regularly by a physician familiar with the physiologic effects of physical measures is an essential part of an adequate treatment program for individuals with rheumatoid arthritis. Too frequently in the clinic we see patients who have had courses of steroid therapy or other medical management without ever having been instructed adequately in a consistent and logical home physical treatment regime. The physician must realize the importance of a comprehensive approach in the treatment of rheumatoid arthritis.

Prescription of Physical Measures.-All too frequently, physicians will send patients to the physical therapist without a prescription other than a request for "physio." The therapist is expected to choose the treatment procedures and to establish his own objectives. In effect, this leads to the practice of medicine by the physical therapist, who has had only limited medical training. A vague or muddled prescription is of little more help to the physical therapist. Another form of vagueness is the "shot-gun" type of prescription which may do nothing more than reveal the physician's lack of knowledge of the proper application of physical agents. No therapeutic measure should be prescribed unless there is a specific reason for its use. Routine prescription of heat, massage, and exercise should be avoided. To obtain the most effective use of physical agents in the treatment of arthritis, the physician should provide the same carefully considered and accurately written prescription for physical procedures that he would for drugs. 
No prescription for physical therapy is complete if it does not provide the physical therapist with the following minimum information ${ }^{3}$ : (1) diagnosis; (2) parts to be treated; (3) specifications regarding procedures or at least the major objectives desired; (4) special instructions regarding anesthetic areas, areas of circulatory embarrassment, removal of dressings, splints, etc.; (5) number and frequency of treatments; (6) date for re-examination by the physician; (7) outline of home instructions, if indicated.

When physical therapy is indicated, any unnecessary delay in starting the treatment may affect the results. In general, the earlier the treatment can be started, the more effective it will be. Also, if the treatment is of real value, it is worth carrying out daily; poor results from physical therapy often occur when treatments are too infrequent. In many cases the treatments should be given twice daily to achieve the most rapid and effective results. Frequent evaluation of the patient's condition is necessary in order to decide whether to continue, discontinue, or alter the treatment program; obviously, therefore, the physician should not lose contact with the patient who is treated by the physical therapist or who is carrying out a home program.

The intelligent use of physical measures and agents requires that the physician have a working knowledge of their physiologic effects. In arthritis, heat is used for its analgesic, muscle relaxing, and vasodilating effects. Massage when properly applied can increase the venous blood flow or lymph flow but is used primarily for its sedative and muscle relaxing effects. Therapeutic exercises employed in arthritis are designed to increase the range of motion of joints or to increase the strength and endurance of muscles. Exercise is the most important part of the physical therapy program.

Listed below are those physical measures most commonly used in the treatment of rheumatoid arthritis along with pertinent remarks regarding their proper application.

Hubbard Tank or Tub Bath:

Employed for general heating

Temperature of water $-100^{\circ}$ to $104^{\circ} \mathrm{F}$.

Duration-15 to 30 minutes

Frequency-daily or every other day

Contraindicated or used with caution in the aged and debilitated

Luminous Bakers:

Used when there is multiple joint involvement; double bakers for general heating

Used at a distance producing a comfortable warmth

Duration-30 minutes

Frequency-one or two times daily

Intensity modified if there is debility, poor circulation or anesthetic area

Infrared Lamps:

Suitable for heating single joints

Used at a distance producing a comfortable warmth

Duration-30 minutes

Frequency -1 or 2 times daily

Intensity modified if there is poor circulation or anesthetic area 


\section{Whirlpool Baths:}

Effective for heating of multiple joints of upper or lower extremities

Water temperature- $105^{\circ}$ to $110^{\circ} \mathrm{F}$.

Duration-30 minutes

Frequency-daily

Water temperature lowered to $100^{\circ} \mathrm{F}$. or less if circulation is impaired

\section{Contrast Baths:}

Used when hands or feet are afflicted

Extremity is placed in hot water $\left(105^{\circ}\right.$ to $110^{\circ} \mathrm{F}$.) for ten minutes, after which it is placed alternately in the cold water $\left(65^{\circ}\right.$ to $70^{\circ} \mathrm{F}$.) for one minute, then for four minutes in the hot water for several alternations, ending in the hot water

Duration- -30 minutes

Frequency - 1 to 2 times daily

Temperature of water modified if painful

Parafin Bath:

Effective method for heating hands and wrists, can be used for feet, can be painted on rounded regions of the body as a pack

Paraffin ( $5 \mathrm{lb}$.) melted in a double boiler, add $1 / 2$ cup of mineral oil, allow to cool until a thin film is formed; hand dipped into paraffin several times until a thick coat is formed, then wrapped in a towel

Duration 20 to 30 minutes

Frequency - 1 or 2 times daily

Contraindicated if there is an open wound

Hot Packs:

May be employed for heating regions of the trunk as well as the joints of the extremities.

Woolen material wrung out of hot water $\left(120^{\circ} \mathrm{F}\right.$.) and placed over affected joints and changed every 10 to 15 minutes; or commerically available silica gel packs wrapped in turkish toweling and applied to the part

Duration-30 to 45 minutes

Frequency -1 or 2 times daily

Contraindicated if there are areas of anesthesia

Short Wave Diathermy, Microwave Diathermy, Ultrasound:

Heat localized regions well but rarely adaptable to safe home use

Pancake, drum, or wrap around coil techniques are the most effective short wave diathermy applications; microwave diathermy applied at 60 to 80 per cent output; ultrasound intensity 0.5 to 1.5 watts/cm. ${ }^{2}$ using stroking technique

Duration-30 minutes for short wave and microwave diathermy; 5 to 10 minutes for ultrasound

Frequency daily

These more potent, deep heating agents should be utilized only by qualitied professional persons

Massage:

Stroking and kneading movements applied to soft tissues adjacent to the involved joints, not to the joint

Mechanical apparatus is not recommended as a substitute

Ordinarily follows use of heat and precedes exercises to increase range of motion 
Intensity of the massage depends on degree of inflammatory reaction in joints and tolerance of the patient

May be employed at home by a member of the patient's family who has been instructed in a few simple massage strokes

Not be employed over areas of infection or malignancy

Not to be used as a reducing procedure, a method for strengthening muscles, or as treatment for psychoneurosis

\section{Therapeutic Exercise:}

Most important single physical measure

Active-assistive type of exercise is used to maintain or increase the range of motion of involved joints

Mobilization exercises should be slow, rhythmical, and through the fullest range possible

Purposeless wiggling of the joints should be avoided

Several short periods of exercise daily are better than one long period

Pain lasting more than two hours after exercise indicates that the exercise was too vigorous

Muscle-setting exercises can be utilized to help preserve the strength of large muscle groups, such as hip and knee extensors

Progressive zesistive exercises are the most effertive in improving strength

Special apparatus may be used, such as weights, exercise table, overhead pulley, shoulder wheel, finger ladder, exercise bicycle, and powder boand

Training in posture may improve body mechanics and reduce strain in involved joints

Gait training with or without crutches or canes may be an important part of the exercise program

The Schedule of Activity.--Every patient with rheumatoid arthritis should be placed on a schedule of activity which provides a balance between rest and exercise. Extra rest prevents and minimizes general fatigue and benefits affected joints. Many times inflammation will subside when joints are rested and properly splinted, and strain is removed. The optimal daily average of rest in bed is 10 to 12 hours, but during exacerbations 12 to 24 hours may be indicated. If the patient is not properly instructed he is likely to assume a flexed position in bed. In order that a patient may rest in correct position a firm flat bed is required. It is not rest or exercise, but rest and exercise.

Instruction of Patient.-Even when the physician does use those measures which have proved to be of value in the management of the patient with rheumatoid arthritis, the patient often will not follow the advice given for home treatment. The physician plays a large role in motivating the patient to carry out an adequate treatment program. It is easier to outline a treatment program than it is to prepare the patient to carry it out. It is probably true that physicians spend more time in evaluating a patient and establishing the direction of treatment than they do in explaining the various treatment procedures, yet the latter is certainly of equal importance. The physician should take the time to explain rheumatoid arthritis as a disease and describe its natural course. He must outline the objectives of the treatment to the patient and thoroughly explain the limitations of the various techniques. If this is done the patient is less likely to entertain hopes of cure or expect reversal of damage already inflicted 
by the disease process. Somehow the physician must help the patient to gain insight into what can be accomplished by physical treatment. The patient must eventually realize that is requires an investment of time and effort on his part and any benefits will be the result of his own hard work.

The method of instruction should take into account individual differences in intelligence. Some patients will learn much more slowly than others; to some of these, the simplest procedures will appear too complicated to follow. Highly intelligent persons will grasp principles and techniques quickly, but since at best, only a certain amount of material can be retained from any one lesson, it is well to instruct the patient two or three times in the actual procedures he will be using at home.

The most successful method is demonstration by actual treatment. If possible, relatives or friends of the patient should watch the procedure and even take part in it, so that both the patient and whoever will be helping him are given the same view and the same understanding of the techniques of treatment. Clearly written instructions will serve to refresh the patient's memory when he is at home and "on his own."

Follow-up Care.-A highly important feature of the home-treatment program is the patient's regular re-evaluation by his physician, so that the treatment may be modified according to changes in the patient's condition. Frequent rechecks on an outpatient basis are necessary, and instruction in modified techniques or new techniques should be given as indicated. Adequate follow-up care may in some cases mean the difference between success and failure of the treatment; moreover, it may provide the key to the difficult problem of motivation of the patient in his long and arduous program of home care.

\section{REFERENCES}

1. Hill, D. F., and Holbrook, W. P.: Prevention and Treatment of Deformities in Rheumatoid Arthritis, J.A.M.A. 142:718, 1950.

2. Treusch, J. V., and Krusen, F. H.: Physical Therapy Applied at Home for Arthritis: A Follow-Up Study With a Supplementary Summary of the Sedimentation Rate of Erythrocytes in 229 Cases of Arthritis, Arch. Int. Med. 72:231, 1943.

3. Martin, G. M.: What Every Physician Should Know About Prescribing Physical Therapy, J.A.M.A. 142:474, 1950 . 